Article

\title{
Protective Immunity Elicited by VP1 Chimeric Antigens of Bacterial Ghosts against Hand-Foot-and-Mouth Disease Virus
}

\author{
Saisai Gong ${ }^{1,+}$, Nan Nan ${ }^{1,+}$, Yakun Sun ${ }^{1,2,+}$, Zhili He ${ }^{1}$, Jiajia Li ${ }^{1}$, Fanghong Chen ${ }^{1}$, Tao Li ${ }^{1}$, \\ Nianzhi Ning ${ }^{1}$, Jianxin Wang ${ }^{1}$, Zhan Li ${ }^{1}$, Deyan Luo ${ }^{1,2, *}$ and Hui Wang ${ }^{1,2, *(D)}$ \\ 1 State Key Laboratory of Pathogen and Biosecurity, Beijing Institute of Microbiology and Epidemiology, \\ 20 Dongda Street, Fengtai District, Beijing 100071, China; gby1738131995@163.com (S.G.); \\ nannan163n@163.com (N.N.); syk_dyx@hotmail.com (Y.S.); lili892017173@163.com (Z.H.); \\ 13024096923@163.com (J.L.); cfh880314@163.com (F.C.); litaobmi@126.com (T.L.); \\ ningnianzhi@163.com (N.N.); jianxinwang1994@163.com (J.W.); yexi19881214@126.com (Z.L.) \\ 2 Department of Microbiology, Anhui Medical University, Hefei 230000, China \\ * Correspondence: wanghui_dyx@hotmail.com (H.W.); ldy612@126.com (D.L.); \\ Tel.: +8610 66948532 (H.W.); Fax: +86 1066948532 (H.W.) \\ + These authors contributed equally to this article.
}

Received: 18 December 2019; Accepted: 28 January 2020; Published: 1 February 2020

check for updates

\begin{abstract}
This study was designed to evaluate the immunogenicity and protective efficacy of two VP1 chimeric antigens of bacterial ghosts. Inoculation of the two VP1 chimeric antigens of bacterial ghosts into BALB/c mice markedly elicited humoral and mucosal immune responses. The specific antibodies induced by the chimeric ghosts protected mice not only against the virus that causes hand-foot-and-mouth disease but also against E. coli O157:H7 bacterial infection. In comparison with the negative control, immunization with the chimeric ghosts protected mice against two LD $_{50}$ hand-foot-and-mouth disease viral infection. In addition, this specific immunity also protected the pups of pregnant mice immunized with the VP1 chimeric antigens of bacterial ghosts against 20 MLD E. coli 0157:H7 infection. Taken together, the results of this study verify for the first time that the VP1 chimeric antigens of bacterial ghosts are target candidates for a new type of vaccine against hand-foot-and-mouth disease. Additionally, this vaccine strategy also elicited a stronger immune response against E. coli O157:H7.
\end{abstract}

Keywords: bacterial ghost; hand, foot, and mouth disease; enterovirus 71; Coxsackie virus

\section{Introduction}

Hand-foot-and-mouth disease (HFMD), a global infectious disease caused by intestinal viruses, has differing outbreak intensities all over the world [1]. Similar to other intestinal pathogens, host resistance to the HFMD virus relies mainly on innate and mucosal immunity $[2,3]$. The mature $B$ lymphocytes that secrete serum-neutralization antibodies is the most important protective response in infected hosts [4]. A vaccine that is nonpathogenic to humans, but effective in stimulating a broad protective immune response, is needed to control HFMD. To develop this type of HFMD vaccine, several research groups are pursuing different strategies, including the development of subunit vaccines and overexpression of protective homologous antigens [5-8]. Another new strategy for developing safe and efficacious vaccines is immunization with bacterial ghosts (BGs) carrying a protective antigen. BGs are produced by the expression of PhiX174 lysis gene $E$ and result in cytoplasmic loss and cellular lysis [9]. BGs maintain the cellular morphology and native antigenic surface structure [10] and possess 
the adjuvant property of bacteria [11,12]. The effects of BG vaccines have been described in various pathogens, such as Vibriocholera [13], Helicobacterpylori [14], and Escherichia coli O157:H7 [15].

HFMD is primarily caused by enterovirus 71 and the Coxsackie virus, both of which are in the Enterovirus genus. Studies have shown that using the VP1 capsid protein of the two viruses as an antigen provides protective immunity against viral infections in a murine model [6,16]. In this study, linear VP1 of the enterovirus 71 (EVP1) and the Coxsackie virus (CVP1) were displayed on the surface of E. coli O157:H7 BGs based on the sandwich vector pSOmpA [17]. The outer membrane protein A (OmpA) of E. coli was used in order to construct a novel candidate vaccine named EVP1 bacterial ghosts (EBGs) and CVP1 bacterial ghosts (CBGs). The immunogenicity, protective ability, and immunologic mechanism of these vaccines in the challenge of the HFMD virus and enterohemorrhagic E. coli O157:H788321 (EHEC) were analyzed in this study.

\section{Materials and Methods}

\subsection{Bacterial Strains, Cells, Plasmids and Virus}

Two E. coli strains were grown in Luria-Bertani (LB) broth or agar (Oxoid LTD, Basingstok, Hampshire, England) supplemented with $100 \mu \mathrm{g} / \mathrm{mL}$ of ampicillin for selection of recombinant plasmid at $37^{\circ} \mathrm{C}$. The bacterial strain E. coli O157:EDL 933 for bacterial ghosts preparation was kept in our lab. The bacterial strain E. coli O157:H788321 for challenge was also kept in our lab. Two wild-type strains ATCC O157:H788321 were purchased from the ATCC center (American Type Culture Collection). A Vero E6 cell line and a HEp-2 cell line were kept in our lab. The expression vector pGEX was purchased from Transgen Inc. (Beijing, China), and display vector PSOMPA and lysis plasmid pLysisE were constructed by our lab [15]. Enterovirus 71 (EV71; GenBank: JQ514785.1) and coxsackievirus B3 (CB3; GenBank: M88483.1), on the basis of which vaccines have been developed, were kept in our lab.

\subsection{Mice}

All animal studies were conducted in accordance with the Beijing Institute of Microbiology and Epidemiology Animal Care and Use Committee guidelines (IACUC 2012). BALB/c wild type mice (5-week-old, weighing 14-16 g) were obtained from our institute's Laboratory Animal Center, Beijing, China. All experimental mice were bred in a specific pathogen-free facility at our institute. Experimental mice were matched for age and sex and cared for according to the guidelines of our institute. Mice were monitored and weighed at least once daily after initiating infection. Recumbent mice, and mice that lost more than $30 \%$ weight, were considered moribund and euthanized.

\subsection{Construction and Preparation of $p O E V P 1$ and $p O C V P 1$}

Full-length open reading frames of the VP1 genes from enterovirus 71 (EVP1) and coxsackievirus B3 (CVP1) were amplified with PCR. The 234-325 amino acids of OmpA amplified from E. coli O157:H7 EDL 933 strain. The PCR primers were designed as follows: EVP1, Forward, 5'-GAATTCGGAGATAGGGTGGC-3', Reverse, 5'- GAGCTCAAGAGTGGTGATCG-3'. CVP1 Forward, 5'- GAATTCGGCCCAGTGGAAGAC-3', Reverse, 5'- GAGCTCAAATGCGCCCGTAT-3'. The two fragments EVP1 and OmpA were digested by EcoRI/SacI (New England Biolabs, Ipswich, MA, USA) and then ligated into the expression vector pGEX between XmaI and $\mathrm{NcoI}$ restriction sites to generate the expression plasmid pOEVP1. The two fragments CVP1 and OmpA were digested by EcoRI/SacI and then ligated into the expression vector PGEX between XmaI and $\mathrm{NcoI}$ restriction sites to generate the expression plasmid pOCVP1. The resulting constructs were transformed into E. coli O157:H7 EDL 933 for bacterial ghosts preparation.

\subsection{Preparation of Bacterial Ghosts and Whole Cells [18]}

The pOEVP1 and pOCVP1 plasmids were transformed into E. coli O157:H7EDL 933 as novel competent cells. LB-medium containing ampicillin was inoculated with E. coli O157:H7 EDL 
933 overnight culture, transformed with the kanamycin resistance and thermolysis plasmid pLysisE to generate BGs (named EBGs and CBGs). E. coli O157:H7 EDL933 strain without pOEVP1 or pOCVP1 plasmid were prepared to BGs as control (named OBGs). In detail, $200 \mathrm{ml}$ of LB-medium containing $100 \mu \mathrm{g} / \mathrm{mL}$ ampicillin and $100 \mu \mathrm{g} / \mathrm{mL}$ kanamycin was inoculated with $5 \mathrm{~mL}$ of each E. coli strain containing plasmids. Grown up to $\mathrm{OD}_{600} 0.3$, the cultures of EBGs and CBGs were induced by isopropyl $\beta$-D-thiogalactopyranoside (IPTG) at a final concentration of $1 \mathrm{mM}$ at $28^{\circ} \mathrm{C}$. The induction of lysis was achieved by shifting the temperature from 28 to $42{ }^{\circ} \mathrm{C}$ when the $\mathrm{OD}_{600}$ reached 0.6 , and the procedure was monitored by the optical densities. The lysis rate based on colony-forming units (CFU) and the morphous of EBGs and CBGs were detected as described previously [19]. Subsequently, BGs vaccine candidates were prepared by the repeated freezing and thawing method to get rid of the surviving bacteria, and then centrifuged $10 \mathrm{~min}$ and washed twice in ice-cold buffer ( $25 \mathrm{mM}$ TBS, Tris-Buffered Saline). These steps may need to be repeated one more time. For long term storage, bacterial ghosts should be lyophilized. The absence of viable cells in the lyophilized samples was determined using colony-forming units.

\subsection{Analysis of Antigens by Fluorescence Activating Cell Sorter (FACS )}

Phosphate buffered solution (PBS) containing mouse anti-EVP1/CVP1 immunoglobin G was incubated with EBGs and CBGs for $1 \mathrm{~h}$, respectively. Then, the FITC-labeled goat anti-mouse IgG was added and incubated for another $1 \mathrm{~h}$ as a second antibody, according to the first antibody, respectively. After processing, the labeled EBGs and CBGs were incubated with $10^{6} \mathrm{HEp}-2$ cells for $4 \mathrm{~h}$. Finally, these cells were harvested and re-suspended with FACS buffer, and $1 \times 10^{5} \mathrm{HEp}-2$ cells were detected by flow cytometry. The OBGs cells were used as control.

\subsection{Analysis of Cytotoxicity by MTT(3-(4,5-dimethyl-2-thiazolyl)-2,5-diphenyl-2-H-tetrazolium bromide)}

Cytotoxicity assays of lyophilized EBGs and CBGs were performed by MTT assay on Vero E6 cells. Serial diluted (1:10) EBGs and CBGs were applied on a Vero E6 cell monolayer and incubated at $37^{\circ} \mathrm{C}$ $\left(\mathrm{CO}_{2}\right)$ for $36 \mathrm{~h}$. Then, $100 \mu \mathrm{L} /$ well $0.5 \mathrm{mg} / \mathrm{ml}$ 3-(4,5-Dimethylthiazol-2-yl)-2,5-diphenyl tetrazolium bromide were added and incubated for another $4 \mathrm{~h}$ to produced formazan crystals. The produced formazan crystals were re-suspended by dimethyl sulphoxide (DMSO, $150 \mu \mathrm{L} /$ well), and the absorbance at $570 \mathrm{~nm}$ was detected. The OBGs and E. coli O157:H7EDL 933 were used as controls.

\subsection{Mice Immunization and Challenge}

All animal studies were conducted in accordance with the Beijing Institute of Microbiology and Epidemiology Animal Care and Use Committee guidelines. Three-week-old male and female BALB/c mice were obtained from our institute's Laboratory Animal Center and divided into groups randomly (20 mice/group). All experimental mice were bred in a specific pathogen-free facility at our institute. Experimental mice were matched for age and sex and cared for according to the guidelines of the institute. The mice were intragastrically administrated with $0.1 \mathrm{mg}$ (corresponding to $10^{8} \mathrm{CFU}$ ) of EBGs and CBGs, which were resuspended in $100 \mu$ of PBS on day 0 for the primary injection and day 14 for boost vaccinations. PBS alone was administrated to the vehicle control group. Twelve mice from each group were then challenged intragastrically 14 days after booster with $20 \mathrm{LD}_{50}\left(2 \times 10^{9} \mathrm{CFU}\right)$ or $50 \mathrm{LD}_{50}\left(5 \times 10^{9} \mathrm{CFU}\right)$ of viable E. coli O157:H788321 strain. Adult mice were resistant to EV71 (or CB3) administrations.

Eight mice from each group were separated and bred for the subsequent study. The pups were used for a virus challenge study. The experimental endpoint was determined, as previously described [20]. One-day-old mice were used to examine the role of maternal antibodies in challenge studies. For maternal immunization, the BALB/c suckling mice possessing passively-transferred maternal EV71 or CB3 antibodies were challenged 1 day after birth with the virus of EV71 and CB3. The mice were observed daily for the occurrence of mortality as the experimental endpoint. 


\subsection{Detection of Specific Antibodies}

The IgA/IgG of serum and irrigating solution specific to OBGs and proteins (intimin, EVP1, and protein CVP1) were measured by enzyme-linked immunosorbent assay. The presence of serum IgG and of the subtypes IgG1, IgG2a, IgG2b, and IgG3 specific to vaccine candidates was determined by indirect ELISA. The BGs (EBGs and CBGs, $0.1 \mathrm{mg} / \mathrm{mL}$ ) and purified proteins (intimin, EVP1, and CVP1, $0.1 \mathrm{mg} / \mathrm{mL}$ ) were coated in 96-well plates overnight at $4{ }^{\circ} \mathrm{C}$, respectively. Serum samples were serially diluted in 2-fold dilutions from 1:10 to 1:20480. The endpoint dilution titer was calculated as the serum dilution resulting in an absorbance reading of 0.2 units above background. Goat anti-mouse IgA-HRP (Sigma, 1:5000) or goat anti-mouse IgG-HRP (Sigma, 1:5000) were used as the detection antibodies. The reactions were developed with TMB $\left(3,3^{\prime}, 5,5^{\prime}\right.$-Tetramethylbenzidine) and stopped with $2 \mathrm{M} \mathrm{H}_{2} \mathrm{SO}_{4}$. The absorbance at $450 \mathrm{~nm}$ was detected.

\subsection{Statistical Analysis}

Statistical analyses were performed using the program Prism 5.0 (GraphPad Software, Inc., LaJolla, California, CA, USA). Values are expressed as mean \pm SD. Data were analyzed by unpaired Student's $t$-test (normal distribution) or one-way ANOVA followed by Dunnett's multiple comparison test. Survival data were analyzed by log-rank tests. Values of $p<0.05$ are considered to be statistically significant.

\section{Results}

\subsection{Preparation and Evaluation of the Chimeric BG Vaccine Candidates}

We constructed specific $B G$ vaccine candidates, EBG and $C B G$, in order to compare the relative roles of these $B G s$ vaccine candidates in inducing an immune response and protective immunity against HFMD virus infection. The expression plasmid pOEVP1 containing $1990 \mathrm{bp}$ of the OEVP1 gene was constructed, and the co-transformation of plasmids pOEVP1 and pLysE successfully generated the vaccine strain of EBGs. The expression plasmid pOCVP1 containing $1950 \mathrm{bp}$ of the OCVP1 gene was constructed, and the co-transformation of plasmids pOCVP1 and p LysE successfully generated the vaccine strain of $C B G s$. The $\mathrm{OD}_{600}$ of both $\mathrm{EBGs}$ and $\mathrm{CBG}$ was reduced constantly after a shift in temperature. The precipitate $1 \mathrm{~h}$ after induction was harvested in order to evaluate the lysis rate, which was counted as $99.99 \% \pm 0.01 \%$ (Figure 1A,B). The morphology of EBGs and CBGs was detected by electron microscopy. Electron microscopy studies showed that the protein VP1-specific transmembrane tunnel structure was not randomly distributed over but was restricted to areas of division sites, predominantly in the middle of the cell or at polar sites [9]. Electron micrographs of a typical bacterial ghost are presented in Figure 1D. To evaluate the safety of the two vaccine candidates, we treated Vero E6 cells with $10 \mathrm{mg}$ of chimeric BGs (equivalent to $1 \times 10^{10} \mathrm{CFU}$ of bacteria) and $1 \times 10^{7}$ CFU of E. coli O157:H7. We found that the pathogenic bacteria killed nearly $100 \%$ of the Vero E6 cells. In contrast, no obvious cytotoxic effects were detected when the Vero E6 cells were treated with $10 \mathrm{mg}$ of EBGs or CBGs ( $p>0.05$; Figure 1C). The outer membrane protein intimin was confirmed in EBGs, CBGs, and OBGs by flow cytometry, but the EVP1 and CVP1 were detected only on the surfaces of EBGs and CBGs (Figure 1E). 
A
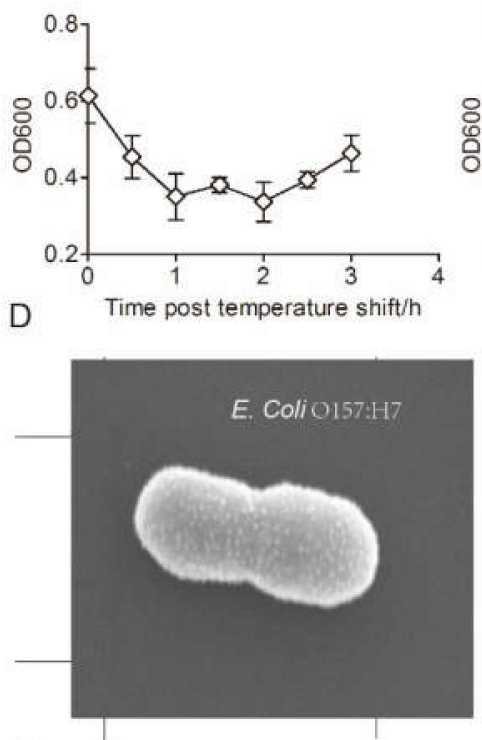

E
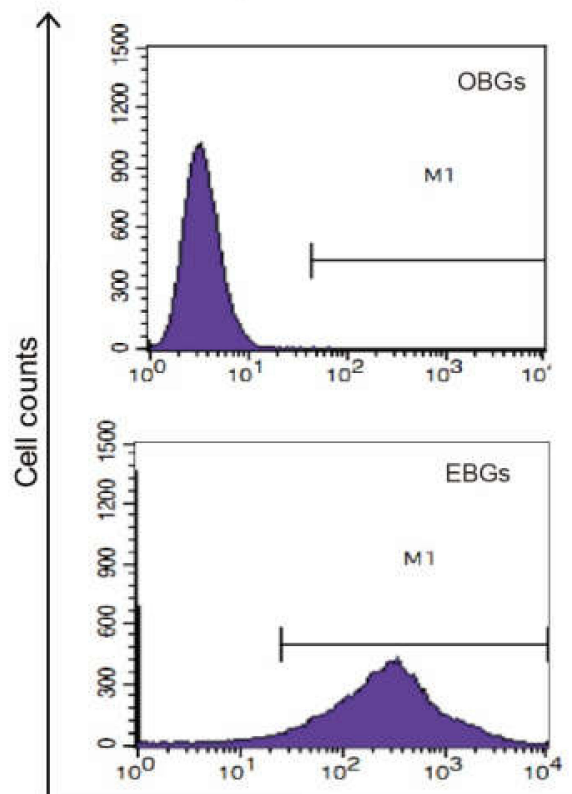

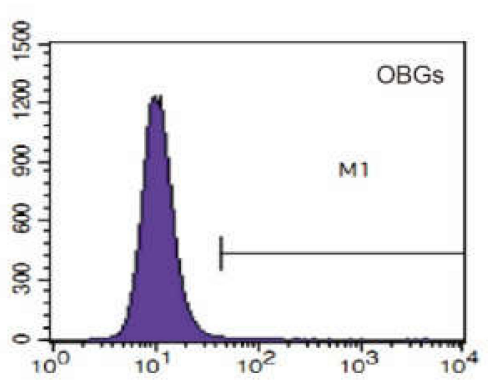

C
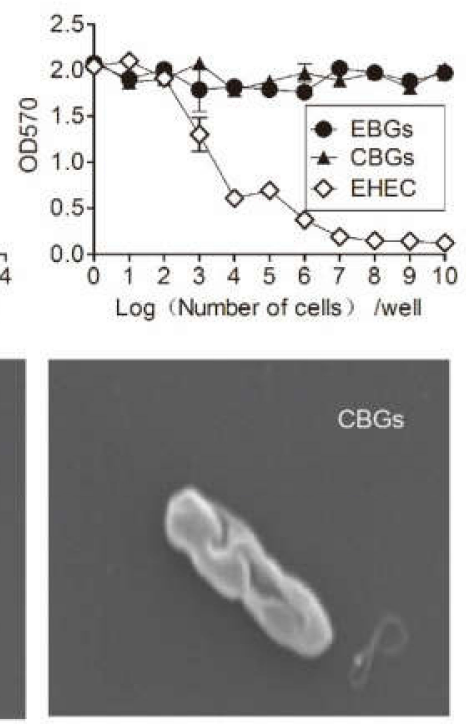

EBGs

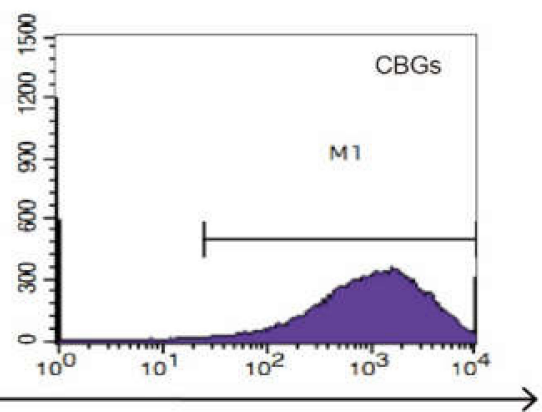

FITC-Ig G

Figure 1. Verification of the bacterial ghost (BG) vaccine candidates and evaluation of the cytotoxicity. (A) Pyrolysisrates of EVP1 bacterial ghosts (EBGs). (B) Pyrolysisrates of EBGs. The $\mathrm{OD}_{600}$ of both EBGs and CVP1 bacterial ghosts (CBGs) were reduced after the shift in temperature. (C) Cytotoxicity analysis of BGs. Serially diluted BGs were incubated with a monolayer of Vero E6 cells for $36 \mathrm{~h}$, and the MTT assay was performed to detect cytotoxicity. (D) The morphology of EBGs and CBGs was assessed by electron microscopy (Scale: E. coli, $12.9 \mathrm{~mm} \times 25 . \mathrm{K} \mathrm{SE}$; EBGs, $12.9 \mathrm{~mm} \times 18 . \mathrm{K} \mathrm{SE}$; CBGs, $12.9 \mathrm{~mm} \times$ 37.K SE). (E) Verification of the surface antigens. Flowcytometry was used to detect $1 \times 10^{5} \mathrm{HEp}-2$ cells. Analysis of E. coli O157:H7 chimeric BG outer membrane proteins, which were identified using an EVP1 or CVP1 antibody. 


\subsection{The Mucosal Immune Response Elicited by Immunization with EBGs or CBGs}

In order to investigate the mucosal immune response induced by the various BGs vaccines, we analyzed the IgA antibody titers specific to BGs, intimin, and VP1 proteins in the serum and irrigating solution of the intestinal tract. Both the sera and irrigating solutions were collected on days $0,7,14,21$, and 28 (3 mice were used for each time point) after the last immunization and were assayed for the presence of IgA antibodies via (enzyme linked immunosorbent assay) ELISA. The results showed that the IgA titers specific to BGs in the sera and irrigating solutions of mice immunized with EBGs and CBGs increased at day 14 and peaked on day 28 (Figure 2). The IgA titers specific to EBGs or CBGs in the hyperimmune sera of immunized mice reached 1:160 and 1:130, respectively (Figure 2A). The IgA titers specific to EBGs or CBGs in the irrigating solutions of immunized mice reached 1:260 and 1:210, respectively (Figure 2B). The IgA titers specific to intimin in the hyperimmune sera of immunized mice reached 1:100 and 1:130, respectively (Figure 2C). The IgA titers specific to intimin in the irrigating solutions of immunized mice reached 1:130 and 1:210 (Figure 2D). The IgA titers specific to the EVP1 protein in the hyper immune sera of mice immunized with EBGs reached 1:130, and no antibody titers were detected in mice immunized with CBGs (Figure 2E). The IgA titers specific to the EVP1 protein in the irrigating solutions of mice immunized with EBGs reached 1:60, and no antibody titers were detected in mice immunized with CBGs (Figure 2F). The IgA titers specific to the CVP1 protein in the hyperimmune sera of mice immunized with CBGs reached 1:60, and no antibody titers were detected in mice immunized with EBGs (Figure 2G). The IgA titers specific to the CVP1 protein in the irrigating solutions of mice immunized with CBGs reached 1:130, and no antibody titers were detected in mice immunized with CBGs (Figure $2 \mathrm{H}$ ). 
A

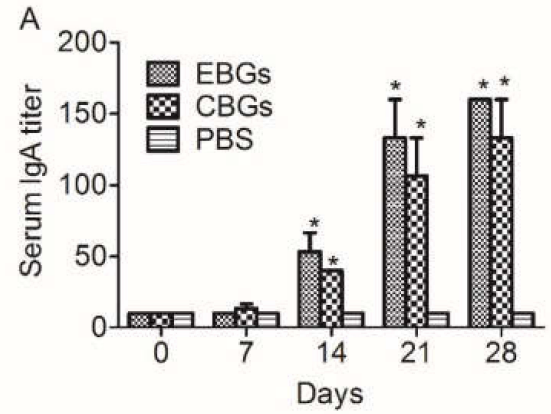

C

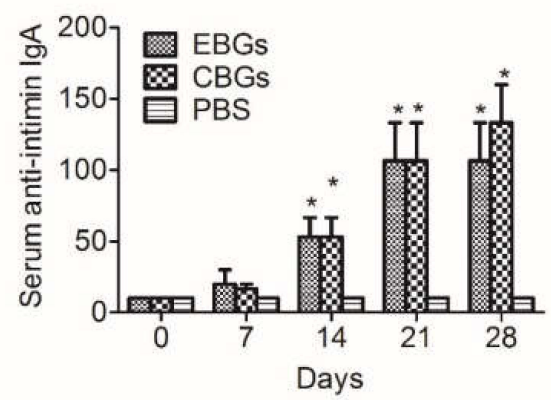

E

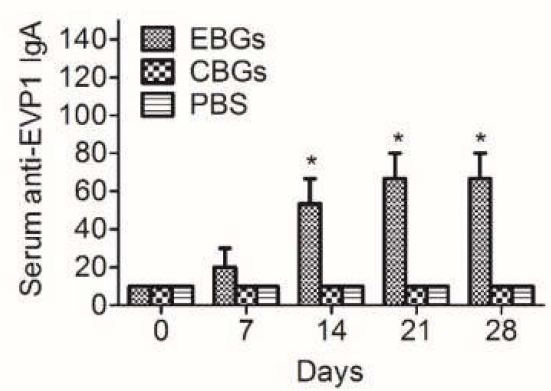

G

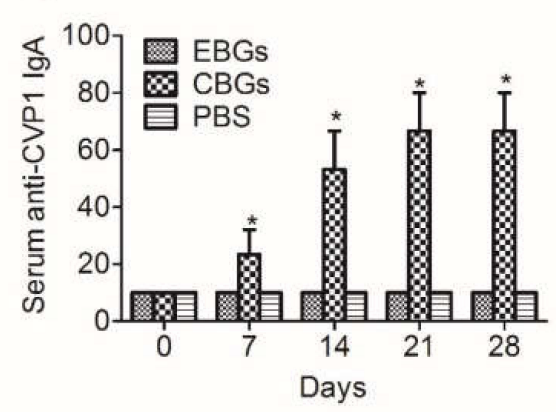

B

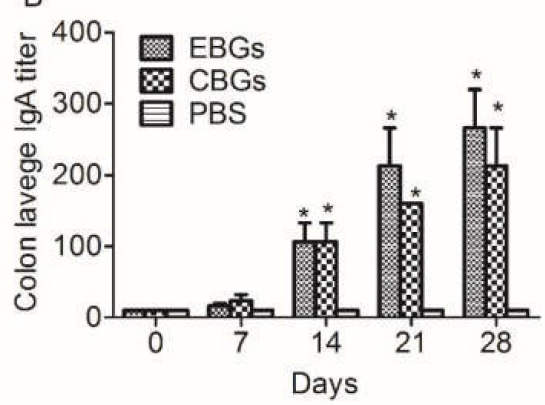

D
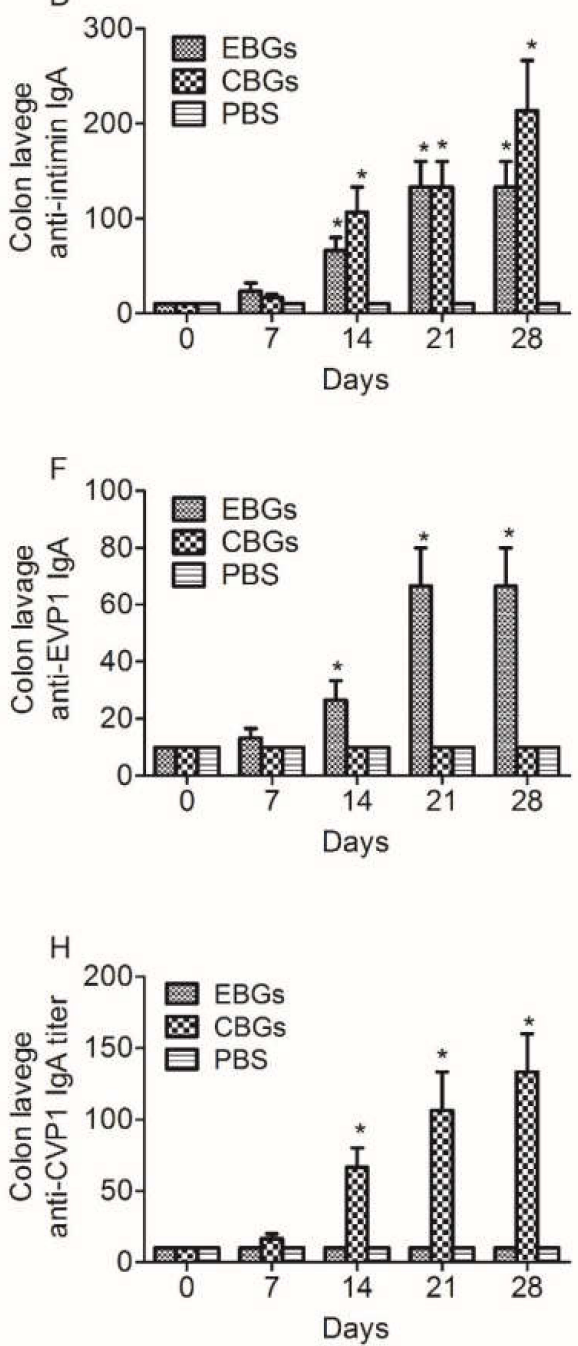

Figure 2. Detection of specific IgA titers in the sera and irrigating solution of immunized mice. Mice were inoculated intragastrically with BGs. Mice that received a PBS injection were negative controls. The IgA levels in the serum and irrigating solution specific to OBGs (E. coli O157:H7 EDL933 strain without pOEVP1 or pOCVP1 plasmids) were measured for specific proteins (intimin, EVP1, and protein CVP1) by enzyme-linked immunosorbent assays. (A) Time course of serum IgA titers specific to OBGs. (B) Time course of irrigating solution IgA titers specific to OBGs. (C) Time course of serum IgA titers specific to intimin. (D) Time course of irrigating solution IgA titers specific to intimin. (E) Time course of serum IgA titers specific to protein EVP1. (F) Time course of irrigating solution IgA titers specific to protein EVP1. (G) Time course of serum IgA titers specific to protein CVP1. (H) Time course of irrigating solution IgA titers specific to protein CVP1. Data are from two independent experiments. ${ }^{*} p<0.01,{ }^{* *} p<0.001$. 


\subsection{The Humoral Immune Response against HFMD Virus Elicited by Immunization with EBGs or CBGs}

In order to further investigate the humoral immune response induced by the various BG vaccines, we analyzed the IgG antibody titers specific to BGs, intimin, and VP1 proteins in the serum and irrigating solution of the intestinal tract. Samples were collected on days 0, 7, 14, 21, and 28 (3 mice were analyzed at each time point after the last immunization and were assayed for the presence of EVP1 and CVP1-specific antibodies by ELISA. Similar to our previous report [15], the specific antibodies induced by CBGs and EBGs increased persistently over time. We detected high levels of specific anti-EBGs and anti-CBGs IgG antibodies in the samples collected from mice immunized with BGs vaccine candidates. No specific antibodies were detected in the PBS-immunized group (Figure 3A,B). The antibodies specific to intimin, one of the most important factors involved in the adhesion of EHEC, were tested. In contrast to PBS, both the CBGs and EBGs were able to induce significant intimin-specific $\operatorname{IgA} / \operatorname{IgG}$ antibodies (Figure 3C,D). The IgG titers specific to the EVP1 protein in the sera of mice immunized with EBGs reached 1:130, and no antibody titers were detected in mice immunized with CBGs (Figure 3E). We did not detect specific IgG antibodies in the irrigating solutions from mice immunized with EBGs and CBGs (Figure 3F). The IgG titers specific to the CVP1 protein in the sera of mice immunized with CBGs reached 1:210, and no antibody titers were detected in mice immunized with EBGs (Figure 3G). We did not detect specific IgG antibodies in the irrigating solutions from mice immunized with EBGs and CBGs (Figure 3H). The analysis of IgG subtypes showed a significant increase in IgG1 in the serum of mice immunized with EBGs or CBGs. The titers reached over 1:1000. The titers of IgG2a and IgG2b were also detected, but only reached about 1:200 (Figure 4).

\subsection{Efficacy of EBGs and CBGs Immunization in Generating Protective Immunity against the HFMD Virus}

While the vaccine approach has great potential for HFMD prevention, immunoprotection against HFMD has not been achieved. In order to study the protective effect of EBGs and CBGs, pups were challenged with an intraperitoneal injection of the EV71 and CB3 viruses. The results showed that EBGs protected mice against $2 \mathrm{LD}_{50}$ of EV71 and CB3 infections (Figure 5A,B). When mice were challenged with $5 \mathrm{LD}_{50}$ EV71, 50\% of the suckling mice immunized with EBGs survived; however, none of the suckling mice immunized with CBGs survived. CBGs provided an $87.5 \%$ protective rate against $2 \mathrm{LD}_{50} \mathrm{CB} 3$ infection and $37.5 \%$ protective rate against $5 \mathrm{LD}_{50} \mathrm{CB} 3$ infection, whereas there were no surviving mice in the $2 \mathrm{LD}_{50}$ EBGs-immunized group for either challenge. There were no survivors in control groups.

\subsection{Efficacy of EBGs and CBGs Immunizations in Generating Cross-Protective Immunity against E. coli 0157:H788321}

EBGs and CBGs not only displayed stronger protection against EV71 and CB3 infections but also displayed cross-protection against E. coli O157:H788321 infection. EBGs provided a similar $(p>0.05)$ protective rate $(14 / 20,70 \%)$ as the CBGs $(15 / 20,75 \%)$ when the challenge dose was 20 LD $_{50}$ (Figure 5C). When the intragastric challenge dose was increased to $50 \mathrm{LD}_{50}$, there were no survivors in either immunized group (data not shown). No mice survived in the PBS-immunized groups when challenged with either the high or low dose. 
A
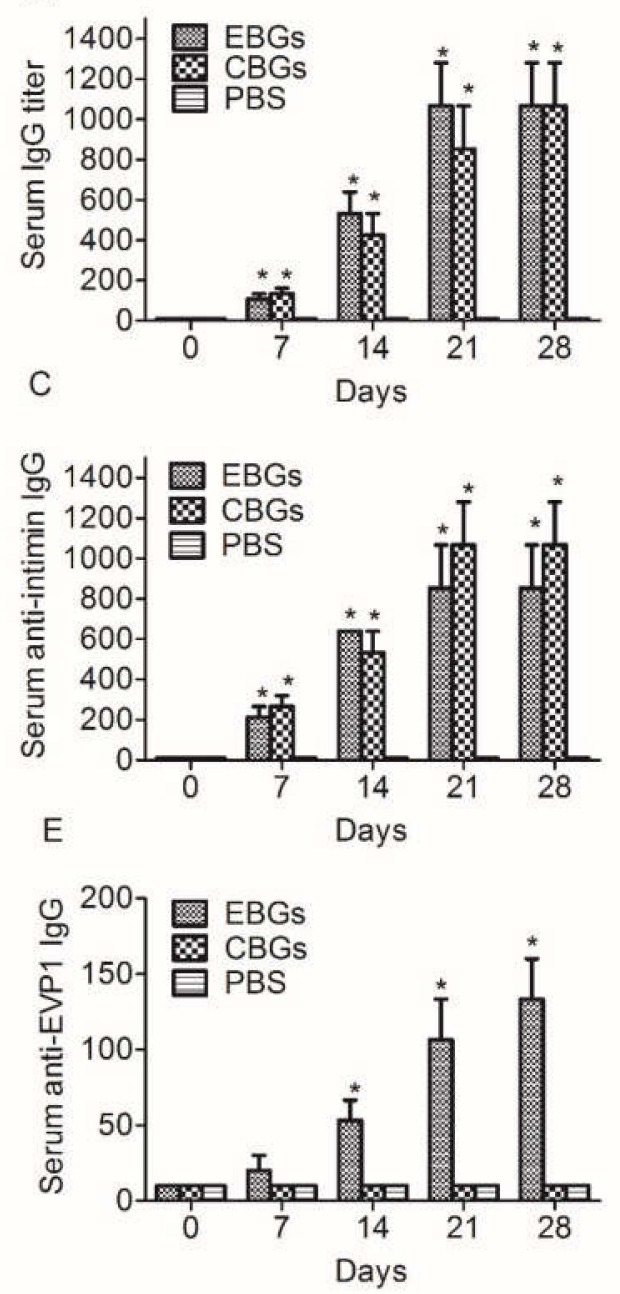

G

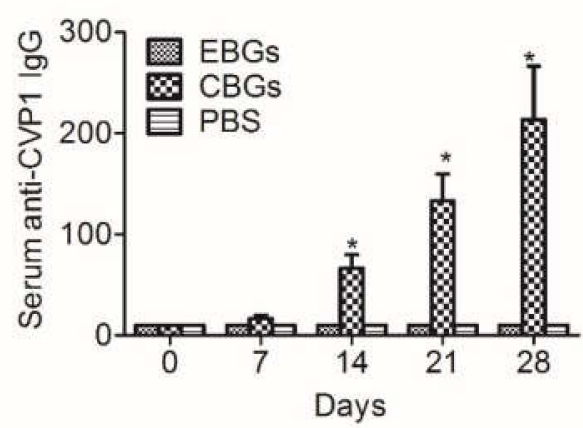

B
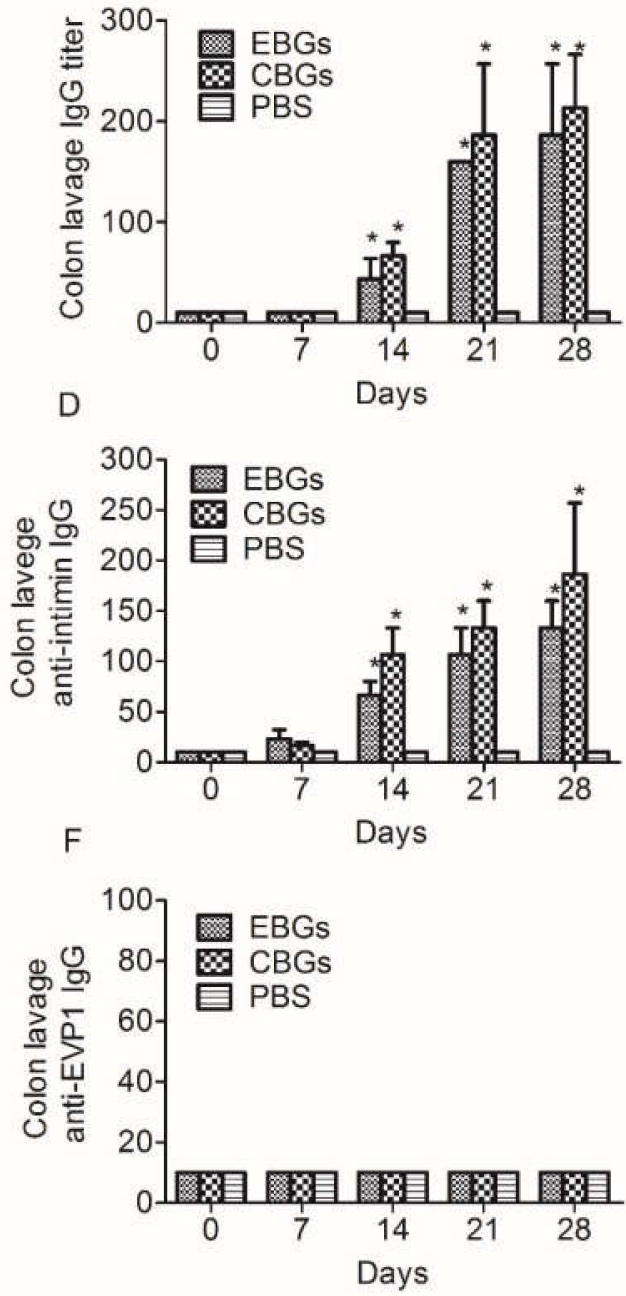

$\mathrm{H}$

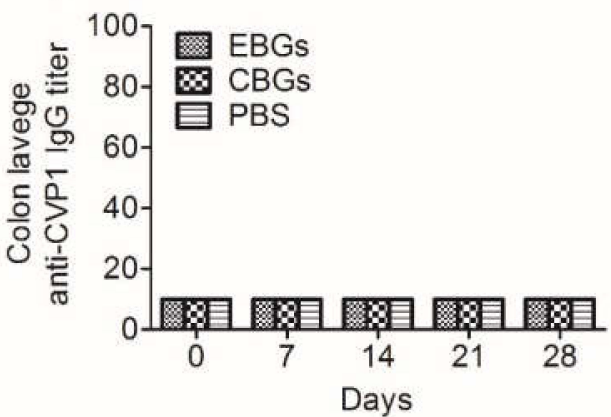

Figure 3. Detection of the specific IgG titers in the sera and irrigating solution of immunized mice. Mice were inoculated intragastrically with BGs. Mice that received a PBS injection were negative controls. The IgG of serum and irrigating solution specific to OBGs and proteins (intimin, EVP1, and protein CVP1) were measured by enzyme-linked immunosorbent assays. (A) Time course of serum IgG titers specific to OBGs. (B) Time course of irrigating solution IgG titers specific to OBGs. (C) Time course of serum IgG titers specific to intimin. (D) Time course of irrigating solution IgG titers specific to intimin. (E) Time course of serum IgG titers specific to protein EVP1. (F) Time course of irrigating solution IgG titers specific to protein EVP1. (G) Time course of serum IgG titers specific to protein CVP1. (H) Time course of irrigating solution IgG titers specific to protein CVP1. Data are from two independent experiments. ${ }^{*} p<0.01$. 


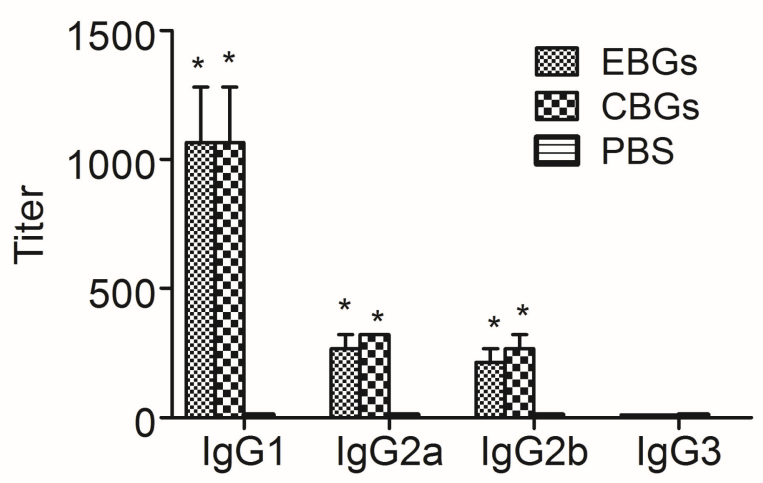

Figure 4. Antibody subtype profiles of mice immunized with various vaccines. Mice were inoculated intragastrically with BGs. Mice that received a PBS injection were negative controls. Two weeks after the last immunization, both the sera and irrigating solution were collected from the experimental mice, and antibody titers were evaluated by an ELISA. Data are from two independent experiments. Each bar represents the mean titers of antibodies in the same group. ${ }^{*} p<0.01$, as compared with the PBS group.
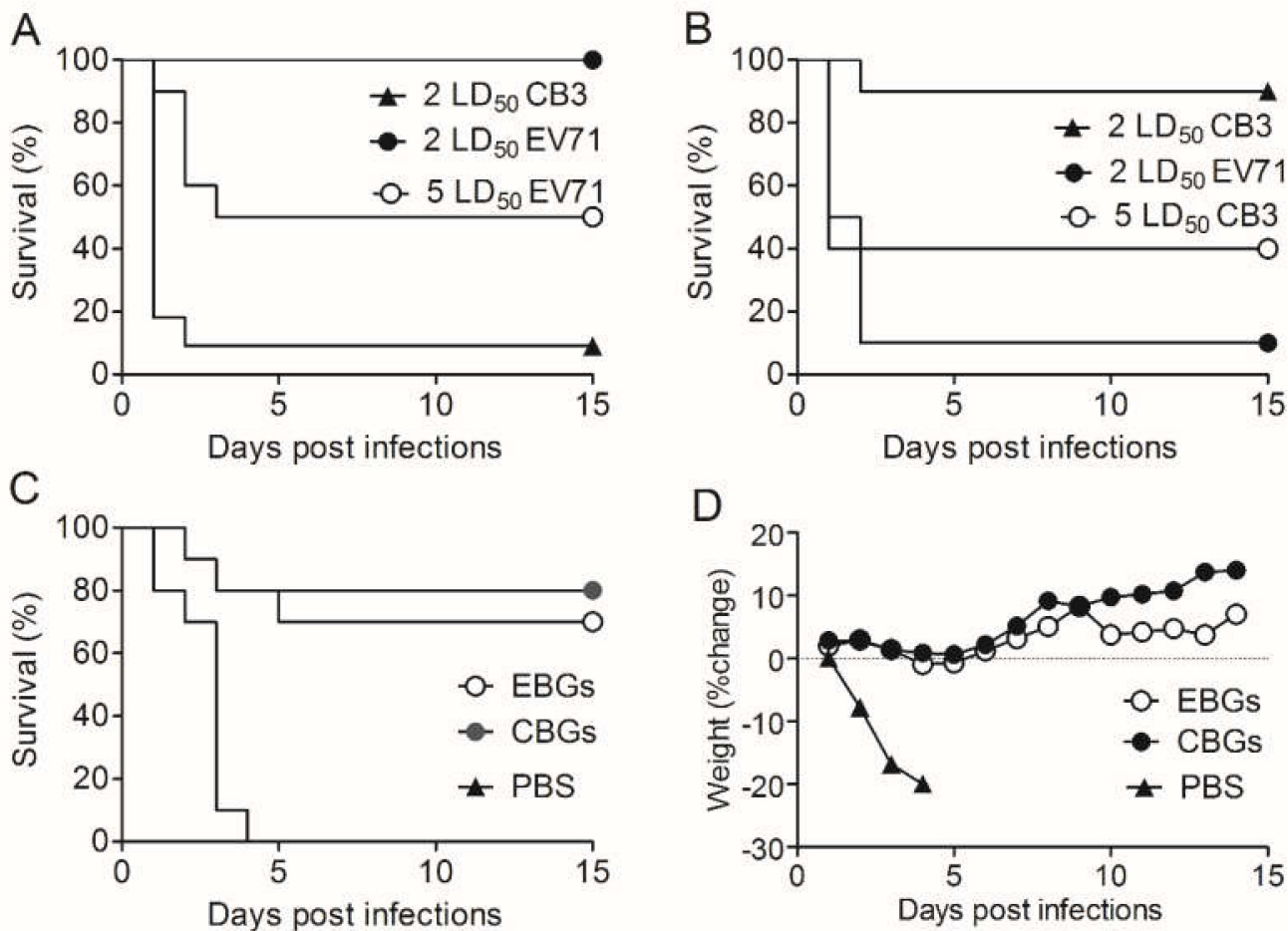

Figure 5. Vaccine candidates protected mice against a lethal dose of toxins. (A) Survival rates of the pups of mice immunized with BGs and challenged with 2 LD $_{50}$ CB3, 2 LD $_{50}$ EV71, or 5 LD $_{50}$ EV71. In comparison with mice immunized with PBS, all vaccine candidates increased the survival rate ( $p<0.001 ; n=20$ mice/group). (B) Survival rates for the pups of mice immunized with BGs and challenged with $2 \mathrm{LD}_{50}$ EV71, $2 \mathrm{LD}_{50} \mathrm{CB} 3$, or $5 \mathrm{LD}_{50} \mathrm{CB}$. In comparison with mice immunized with PBS, all vaccine candidates increased the survival rate $(p<0.001 ; n=20$ mice/group). (C) Survival rate $(D)$ body weight changes for wild-type mice immunized with BGs and challenged with $20 \mathrm{LD}_{50}$ $\left(2 \times 10^{9} \mathrm{CFU}\right)$ of viable E. coli O157:H788321 strain. In comparison with mice immunized with PBS, all vaccine candidates increased the survival rate $(p<0.001 ; n=20$ mice/group). Similar results were observed in two independent experiments. Survival data were analyzed by log-rank tests. Data are from two independent experiments. 


\section{Discussion}

In recent years, there has been an increase in the occurrence of HFMD, which has no effective treatment. Disease prevention is a promising approach to defend against HFMD virus infection, which deserves further exploration [16]. As mentioned previously, capsid protein VP1 is a major virulence determinant of the HFMD virus. A number of studies have demonstrated that antibodies induced by VP1-based vaccines can efficiently block viral replication in tissue [21,22]. Although these VP1 protein vaccine candidates can be produced easily, they are less immunogenic and require a delivery system or the addition of adjuvants. BGs are produced by the expression of the cloned PhiX174 gene E in Gram-negative bacteria [23], which can be used as a delivery system for foreign subunit antigens. It has been demonstrated that foreign antigens presented by BGs to the immune system can induce strong immune responses [14,24,25].

Therefore, we designed two VP1 chimeric antigens of BGs as vaccine candidates in this study. We immunized mice with the two chimeric BGs and challenged them with E. coli O157:H7. We also challenged suckling mice with EV71 and CB3 virus separately. In this study, it was verified for the first time that two BG vaccines based on the VP1 proteins induced significant mucosal and humoral immunity. We detected high levels of specific IgG and IgA titers 7 days after the first immunization, and the titers were much higher on day 7 after a booster. From these results, we concluded that both CBGs and EBGs were highly immunogenic for mice. To investigate which subtype was predominant in mice immunized with the vaccine candidates, the IgG subtypes were assayed by ELISA. We found a significant increase in the absorbance for antigen-specific IgG1, IgG2a, and IgG2b, but not IgG3.

Recombinant VP1 protein formulated with complete Freund's adjuvant can elicit neutralizing antibody responses in mice. Synthetic immunogens based on the neutralization epitopes of EV71 also require complete Freund's adjuvant, which is not acceptable for use in humans [16]. Vaccination with DNA plasmid constructs encoding VP1 resulted in low neutralizing responses. Vaccination of neonatal mice with an adenovirus vector expressing a conserved neutralization epitope conferred protection against lethal EV71 challenge. Thus, delivery systems are very important for HFMD virus vaccine development. Here, we selected BGs as a delivery system for our vaccine candidates. In order to evaluate the protection conferred by the vaccine candidates, the newborn mice were challenged with an intraperitoneal injection of EV71 and CB3 virus separately. We found that the two vaccine candidates protected mice against HFVD virus infection.

The immunized adult mice were intragastrically challenged with E. coli O157:H7. We found that the two vaccine candidates also protected mice against $E$. coli infection. Intimin protein, a membrane component on the surface of E. coli O157:H7, is an important factor for bacterial attaching/effacing (A/E), and the levels of intimin specific antibodies have been demonstrated to be associated with protection against this bacterium [26,27]. In our study, we evaluated high levels of anti-intimin antibody responses. Therefore, the novel BG vaccine candidates have the potential to provide protection against the adhesion of bacteria. We did not find a significant difference in the protection of CBG-and EBG-immunized mice challenged with E. coli.

To summarize, our chimeric BGs vaccine candidates elicit a higher mucosal immune response and provide greater protection for the host against HFMD. Our data decisively demonstrated that our vaccine candidates also conferred cross-protection against E. coli O157:H7, indicating that the BGs can be used as a relatively efficacious vector for vaccine development against HFMD.

Author Contributions: All authors discussed the results and implications of the manuscript. H.W. and D.L. conceived the study, supervised the project, analyzed the data, and wrote the paper. S.G., N.N., Z.H., J.L., Y.S., T.L., F.C., J.W. and Z.L. performed the experiments and analyzed the data. D.L. advised on statistical evaluations. S.G., N.N. and Y.S. contributed equally to this work. All authors have read and agreed to the published version of the manuscript.

Funding: National Key Research Project: 2018ZX10101003-005-001; National Natural Science Foundation of China: 31870156; National Key Lab Research program: SKLPBS1811 
Acknowledgments: All animal studies were conducted in accordance with the Beijing Institute of Microbiology and Epidemiology Animal Care and Use Committee guidelines. We thank all employees of the Beijing Institute of Microbiology and Epidemiology Animal Facilities for dedicated breeding and care of the mice used in these studies.

Conflicts of Interest: The authors have no financial conflicts of interest.

\section{References}

1. Wang, B.; Li, J.; Wang, Y.; Du, N.; Sun, L.; Xiao, H.; Zhao, Y.; Bao, W.; Zhang, W. Understanding the epidemiological characteristics of EV71 and CVA16 infection to aid the diagnosis and treatment of hand, foot, and mouth disease. J. Med Virol. 2019, 91, 201-207. [CrossRef] [PubMed]

2. Pathinayake, P.S.; Hsu, A.C.; Wark, P.A. Innate immunity and immune evasion by enterovirus 71 . Viruses 2015, 7, 6613-6630. [CrossRef] [PubMed]

3. Lin, Y.L.; Cheng, P.Y.; Chin, C.L.; Huang, L.M.; Lin, S.Y.; Chiang, B.L. Fibroblast-stimulating lipopeptide-1 as a potential mucosal adjuvant enhances mucosal and systemic immune responses to enterovirus 71 vaccine. Vaccine 2018, 36, 4331-4338. [CrossRef]

4. Wang, L.C.; Kao, C.M.; Ling, P.; Su, I.J.; Chang, T.M.; Chen, S.H. CD4 T-cell-independent antibody response reduces enterovirus 71 lethality in mice by decreasing tissue viral loads. Clin. Dev. Immunol. 2012, 2012, 580696. [CrossRef]

5. Tung, W.S.; Bakar, S.A.; Sekawi, Z.; Rosli, R. DNA vaccine constructs against enterovirus 71 elicit immune response in mice. Genet. Vaccines Ther. 2007, 5, 6. [CrossRef]

6. Crawford, N.W.; Graham, S.M. EV71 vaccine: protection from a previously neglected disease. Lancet 2013, 381, 1968-1970. [CrossRef]

7. Park, K.B.; Lim, B.K.; Ye, M.B.; Chung, S.Y.; Nam, J.H. A peptide vaccine based on a B-cell epitope on the VP1 protein of enterovirus 70 induces a strong antibody response. Acta. Virologica. 2012, 56, 337-342. [CrossRef]

8. Xu, L.; He, D.; Li, Z.; Zheng, J.; Yang, L.; Yu, M.; Yu, H.; Chen, Y.; Que, Y.; Shih, J.W.; et al. Protection against lethal enterovirus 71 challenge in mice by a recombinant vaccine candidate containing a broadly cross-neutralizing epitope within the VP2 EF loop. Theranostics 2014, 4, 498-513. [CrossRef]

9. Witte, A.; Wanner, G.; Sulzner, M.; Lubitz, W. Dynamics of PhiX174 protein E-mediated lysis of Escherichia coli. Arch. Microbiol. 1992, 157, 381-388. [CrossRef]

10. Witte, A.; Lubitz, W. Biochemical characterization of phi X174-protein-E-mediated lysis of Escherichia coli. Eur. J. Biochem. 1989, 180, 393-398. [CrossRef]

11. Riedmann, E.M.; Kyd, J.M.; Cripps, A.W.; Lubitz, W. Bacterial ghosts as adjuvant particles. Expert Rev. Vaccines 2007, 6, 241-253. [CrossRef]

12. Jiao, H.; Yang, H.; Zhao, D.; Chen, J.; Zhang, Q.; Liang, J.; Yin, Y.; Kong, G.; Li, G. Design and immune characterization of a novel Neisseria gonorrhoeae DNA vaccine using ghosts as vector and adjuvant. Vaccine 2018, 36, 4532-4539. [CrossRef]

13. Eko, F.O.; Schukovskaya, T.; Lotzmanova, E.Y.; Firstova, V.V.; Emalyanova, N.V.; Klueva, S.N.; Kravtzov, A.L.; Livanova, L.F.; Kutyrev, V.V.; Igietseme, J.U.; et al. Evaluation of the protective efficacy of Vibrio cholerae ghost (VCG) candidate vaccines in rabbits. Vaccine 2003, 21, 3663-3674. [CrossRef]

14. Panthel, K.; Jechlinger, W.; Matis, A.; Rohde, M.; Szostak, M.; Lubitz, W.; Haas, R. Generation of Helicobacter pylori ghosts by PhiX protein E-mediated inactivation and their evaluation as vaccine candidates. Infect. Immun. 2003, 71, 109-116. [CrossRef]

15. Cai, K.; Tu, W.; Liu, Y.; Li, T.; Wang, H. Novel fusion antigen displayed-bacterial ghosts vaccine candidate against infection of Escherichia coli O157:H7. Sci. Rep. 2015, 5, 17479. [CrossRef]

16. Chong, P.; Liu, C.C.; Chow, Y.H.; Chou, A.H.; Klein, M. Review of enterovirus 71 vaccines. Clin. Infect. Dis.: Off. Publ. Infect. Dis. Soc. Am. 2015, 60, 797-803. [CrossRef]

17. Pistor, S.; Hobom, G. Expression of viral hemagglutinin on the surface of E. coli. Klin. Wochenschr. 1988, 66, 110-116. [CrossRef]

18. Mader, H.J.; Szostak, M.P.; Hensel, A.; Lubitz, W.; Haslberger, A.G. Endotoxicity does not limit the use of bacterial ghosts as candidate vaccines. Vaccine 1997, 15, 195-202. [CrossRef] 
19. Cai, K.; Gao, X.; Li, T.; Hou, X.; Wang, Q.; Liu, H.; Xiao, L.; Tu, W.; Liu, Y.; Shi, J.; et al. Intragastric immunization of mice with enterohemorrhagic Escherichia coli O157:H7 bacterial ghosts reduces mortality and shedding and induces a Th2-type dominated mixed immune response. Can. J. Microbiol. 2010, 56, 389-398. [CrossRef]

20. Fanghong, C.; Xiong, L.; Nan, N.; Zhan, L.; Nianzhi, N.; Deyan, L.; Hui, W. VP1 of Enterovirus 71 Protects Mice Against Enterovirus 71 and Coxsackievirus B3 in Lethal Challenge Experiment. Front. Immunol 2019, 10, 2564.

21. Wu, C.Y.; Yu, S.L.; Chen, Y.T.; Chen, Y.H.; Hsiao, P.W.; Chow, Y.H.; Chen, J.R. The mature EV71 virion induced a broadly cross-neutralizing VP1 antibody against subtypes of the EV71 virus. PLoS One 2019, 14, e0210553. [CrossRef]

22. Zheng, H.; Wang, J.; Li, B.; Guo, L.; Li, H.; Song, J.; Yang, Z.; Li, H.; Fan, H.; Huang, X.; et al. Correction: A Novel Neutralizing Antibody Specific to the DE Loop of VP1 Can Inhibit EV-D68 Infection in Mice. J. Immunol. 2019, 202, 1905-1906. [CrossRef]

23. Walcher, P.; Mayr, U.B.; Azimpour-Tabrizi, C.; Eko, F.O.; Jechlinger, W.; Mayrhofer, P.; Alefantis, T.; Mujer, C.V.; DelVecchio, V.G.; Lubitz, W. Antigen discovery and delivery of subunit vaccines by nonliving bacterial ghost vectors. Expert Rev. Vaccines 2004, 3, 681-691. [CrossRef]

24. Jechlinger, W.; Haller, C.; Resch, S.; Hofmann, A.; Szostak, M.P.; Lubitz, W. Comparative immunogenicity of the hepatitis B virus core 149 antigen displayed on the inner and outer membrane of bacterial ghosts. Vaccine 2005, 23, 3609-3617. [CrossRef]

25. Ebensen, T.; Paukner, S.; Link, C.; Kudela, P.; de Domenico, C.; Lubitz, W.; Guzman, C.A. Bacterial ghosts are an efficient delivery system for DNA vaccines. J. Immunol. 2004, 172, 6858-6865. [CrossRef]

26. Klose, M.; Jahnig, F.; Hindennach, I.; Henning, U. Restoration of membrane incorporation of an Escherichia coli outer membrane protein (OmpA) defective in membrane insertion. J. Biol. Chem. 1989, 264, 21842-21847.

27. Dornmair, K.; Kiefer, H.; Jahnig, F. Refolding of an integral membrane protein. OmpA of Escherichia coli. J. Biol. Chem. 1990, 265, 18907-18911.

(C) 2020 by the authors. Licensee MDPI, Basel, Switzerland. This article is an open access article distributed under the terms and conditions of the Creative Commons Attribution (CC BY) license (http://creativecommons.org/licenses/by/4.0/). 